\title{
Incertidumbre ante la enfermedad COVID-19 en personal de enfermería de hospitales de Morelia, México
}

\section{Uncertainty about COVID-19 disease among nurses in hospitals in Morelia, Mexico}

\section{Incerteza sobre a doença COVID-19 em enfermeiras hospitalares em Morelia, México}

\author{
Ma. de Jesús Ruiz-Recéndiz ${ }^{1 *}$ \\ https://orcid.org/0000-0002-7979-4215 \\ Vanesa Jiménez-Arroyo ${ }^{2}$ \\ (D) https://orcid.org/0000-0003-3413-3947 \\ Mayra Itzel Huerta-Baltazar ${ }^{3}$ \\ (iD) https://orcid.org/0000-0003-0908-424X \\ Ma. Lilia Alicia Alcántar-Zavala ${ }^{4}$ \\ (iD) https://orcid.org/0000-0003-1528-3077 \\ José Manuel Herrera-Paredes 5 \\ Dttps://orcid.org/0000-0001-7631-5904 \\ Graciela González-Villegas ${ }^{6}$ \\ Dttps://orcid.org/0000-0001-7764-2245
}

1. Doctora en Ciencias de Enfermería, Profesor e investigador, Universidad Michoacana de San Nicolas de Hidalgo. Morelia, Michoacán, México.

2. Doctora en Ciencias de Enfermería, Profesor e investigador, Universidad Michoacana de San Nicolas de Hidalgo. Morelia, Michoacán, México.

3. Doctora en Ciencias de Enfermería, Profesor e investigador, Universidad Michoacana de San Nicolas de Hidalgo. Morelia, Michoacán, México.

4. Doctora en Ciencias de Enfermería, Profesor e investigador, Universidad Michoacana de San Nicolas de Hidalgo. Morelia, Michoacán, México.

5. Doctor en Ciencias de Enfermería, Profesor e investigador, Universidad de Guanajuato. Celaya-Salvatierra, Guanajuato, México.

6. Maestra en Enfermería. Profesor de asignatura. Universidad Michoacana de San Nicolas de Hidalgo. Morelia, Michoacán, México.

*Autor para correspondencia: madejesus.ruiz@umich.mx

Recibido: $31 / 10 / 2020$

Aceptado: 13/11/2021 


\title{
Resumen
}

Introducción: La irrupción de enfermedades emergentes como la COVID-19 provoca una serie de alteraciones en las personas, incluyendo al personal de enfermería, quienes se encuentran en la primera línea de atención. Algunas afectaciones incluyen la presencia de ansiedad, depresión, estrés, insomnio y angustia. Objetivo: Evaluar el nivel de incertidumbre ante la enfermedad COVID-19 en personal de enfermería de segundo nivel de atención en Morelia, Michoacán, México. Metodología: Estudio descriptivo y transversal, en muestra de 160 enfermeras/os de hospitales públicos o privados de segundo nivel de atención, muestreo no probabilístico a conveniencia contactados vía Facebook. Se incluyeron enfermeras en atención directa a pacientes, utilizando Escala de Incertidumbre ante la Enfermedad en versión comunitaria modificada y cédula de datos sociodemográficos y laborales. Se empleó estadística descriptiva y chi cuadrada. Se respetó el anonimato de los participantes. Resultados. La mayoría de los participantes fueron mujeres (87.5\%), licenciadas en enfermería (63.1\%), edad promedio de 36.7 años $(D E=8.5)$ y $36.9 \%$ habían tenido contacto con pacientes COVID-19. La incertidumbre total fue moderada en $56.9 \%$ y en personal de enfermería en contacto con pacientes COVID-19 fue 64.8\%. Se encontró relación estadísticamente significativa $(p=\leq 0.05)$ entre incertidumbre y sexo, escolaridad; capacitación en COVID-19 y uso de equipo de protección personal; disponibilidad de careta y equipo de protección personal; tomar curso de COVID-19 y tener contacto con pacientes COVID-19. Conclusiones. Existió incertidumbre moderada en el personal de enfermería de hospitales de segundo nivel de atención, con diferencias en variables personales, de capacitación y disponibilidad de material y equipo.

Palabras clave: Incertidumbre; Enfermeras; Infecciones por coronavirus; Pacientes; COVID-19 (DeCS).

\begin{abstract}
Introduction: The outbreak of emerging diseases such as COVID-19 has caused a series of alterations in people, including nurses, who are in the first line of care. Some of the effects include the presence of anxiety, depression, stress, insomnia, and anguish. Objective: Evaluate the level of uncertainty about COVID-19 disease in second-level nursing staff in Morelia, Michoacán, Mexico. Methodology: Descriptive and crosssectional study, in a sample of 160 nurses from public and private second-level care hospitals, nonprobabilistic convenience sampling contacted via Facebook. Nurses in direct patient care were included, using the modified community version of the Illness Uncertainty Scale and a sociodemographic and occupational data questionnaire. Descriptive statistics and chi-square were used. The anonymity of the participants was respected. Results. Most of the participants were women (87.5\%), licensed nurses (63.1\%), mean age 36.7 years $(S D=8.5)$ and $36.9 \%$ had been in contact with COVID-19 patients. Total uncertainty was moderate in $56.9 \%$ and in nursing personnel in contact with COVID-19 patients it was $64.8 \%$. A statistically significant relationship ( $p=\leq 0.05$ ) was found between uncertainty and sex, schooling; COVID-19 training and use of personal protective equipment; availability of face shield and personal protective equipment; have taken COVID-19 course and have had contact with COVID-19 patients. Conclusions. There was moderate uncertainty in the nursing personnel working at second-level care hospitals, with differences in personal variables, training and availability of material and equipment.
\end{abstract}

Keywords: Uncertainty; Nurses; Coronavirus infections; Patients; COVID-19 (DeCS).

\begin{abstract}
Abstrato
Introdução: A irrupção de doenças emergentes como a COVID-19 causa uma série de alterações nas pessoas, incluindo enfermeiras, que estão na primeira linha de atendimento. Alguns dos efeitos incluem a presença de ansiedade, depressão, estresse, insônia e angústia. Objetivo: Avaliar o nível de incerteza sobre
\end{abstract}


a doença COVID-19 no pessoal de enfermagem de segundo nível em Morelia, Michoacán, México. Metodologia: Estudo descritivo e transversal, em uma amostra de 160 enfermeiros de hospitais públicos ou privados de segundo nível de assistência, amostra de conveniência não-probabilística contatados via Facebook. Foram incluídos enfermeiros no atendimento direto a pacientes, utilizando a versão modificada da Escala de Incerteza de Doença e um questionário de dados sociodemográficos e ocupacionais. Foram usadas estatísticas descritivas e o qui-quadrado. $\mathrm{O}$ anonimato dos participantes foi respeitado. Resultados. A maioria dos participantes eram mulheres (87,5\%), enfermeiras licenciadas (63,1\%), idade média de 36,7 anos $(S D=8,5)$ e $36,9 \%$ tinham estado em contato com pacientes da COVID-19. A incerteza total foi moderada em $56,9 \%$ e no pessoal de enfermagem em contato com os pacientes da COVID-19 foi de $64,8 \%$. Foi encontrada uma relação estatisticamente significativa $(p=\leq 0,05)$ entre incerteza e sexo, escolaridade; treinamento COVID-19 e uso de equipamento de proteção pessoal; disponibilidade de máscara facial e equipamento de proteção pessoal; fazer o curso COVID-19 e ter contato com pacientes COVID-19. Conclusões. Houve incerteza moderada na equipe de enfermagem dos hospitais de segundo nível, com diferenças nas variáveis pessoais, treinamento e disponibilidade de material e equipamento.

Palavras-chave: Incerteza; enfermeira/o; infecções devido ao Coronavírus; pacientes (DeCS).

\section{Introducción}

La enfermedad COVID-19 causada por el nuevo coronavirus SARS-CoV-2 fue considerada pandemia por la Organización Mundial de la Salud (OMS) el 11 de marzo del 2020(1). El personal de enfermería se encuentra en primera línea del cuidado de los pacientes diagnosticados con COVID-19, quienes realizan un cuidado con calidad que garantiza la seguridad del paciente ${ }^{(2)}$. Para lograr lo anterior, los sistemas de salud requieren la disponibilidad de suficiente personal de enfermería, y durante esta pandemia se mostró la carencia de recursos humanos en enfermería en el mundo. Cifras reportan la existencia de 27.9 millones de enfermeras (os), con una relación de 4.6 enfermeras por médicos en países europeos ${ }^{(3)}$, mientras que en México se estima una tasa de 2.5 enfermeras (os) por 100000 habitantes, en tanto que en el Estado de Michoacán de Ocampo en México, la tasa de 1.7 de personal de enfermería por 100000 habitantes es la más baja de todo el país ${ }^{(4)}$. Estos datos resultan de gran importancia, dado que la escasez de recursos humanos en enfermería contribuye a aumentar el impacto negativo para la salud de las enfermeras (os) con factores psicosociales como estrés, dificultad para quedarse dormido o despertar durante la noche ${ }^{(5)}$, dolor o insatisfacción por la pérdida de un paciente ${ }^{(6)}$; además de factores laborales como la exposición a patógenos, estigma y violencia física y psicológica ${ }^{(7)}$, lo que se acrecentó durante la pandemia por COVID-19. 
Aunque se reconoce que el estrés psicológico y las emociones negativas experimentadas durante una epidemia son esperables, existen numerosos estresores generales y específicos que las enfermeras (os) enfrentan en las crisis de salud y que les provoca incertidumbre.

Se consideran como estresores específicos al riesgo de infectarse a sí mismo, a la familia o a otros, o la interpretación errónea de síntomas de gripe con la enfermedad COVID-19(8-9); así como el miedo a morir o al aislamiento social por la asociación con la enfermedad(10).

Existen además estresores de tipo institucional como la estigmatización de trabajar con pacientes con COVID-19, molestias por el uso de Equipos de Protección Personal (EPP), insuficiente capacitación de EPP o escasez del mismo, inexperiencia o pocas enfermeras (os) y la percepción de apoyo limitado de las autoridades de salud durante la pandemia ${ }^{(8-14)}$. Adicionalmente, las enfermeras (os) pueden experimentar frustración ante la muerte de los pacientes, muchas veces inevitable, o incluso padecer riesgo de agotamiento y fatiga por compasión de los pacientes que cuidan en las Unidades de Cuidados Intensivos $(\mathrm{UCl}){ }^{(9-10,15-17)}$.

Con base en lo anterior, existen múltiples elementos durante la pandemia que provocan en las enfermeras (os) estrés psicológico, disminución del apetito, fatiga, dificultad para dormir, nerviosismo, llanto frecuente ${ }^{(8-9)}$ e incluso pensamientos suicidas e incertidumbre cuando están en atención directa de pacientes con COVID-19 por sentirse aisladas y vulnerables a infectarse ${ }^{(18-23)}$.

La incertidumbre, de acuerdo con la teoría de Merle Mishel, se define como la incapacidad de determinar el significado de los hechos que guardan relación con la enfermedad, y durante la pandemia por la enfermedad COVID-19, las enfermeras (OS) se enfrentan a situaciones laborales, familiares y personales desconocidas derivadas de la pandemia que les impide determinar el resultado que tendrá para su vida ${ }^{(24)}$. Por tanto, es importante investigar la variable incertidumbre desde el sustento teórico de Michel para crear evidencia empírica que fortalezca la disciplina de enfermería. Lamentablemente no se localizaron estudios con esta temática, por lo que se planteó como objetivo evaluar el nivel de incertidumbre ante la enfermedad 
COVID-19 en el personal de enfermería de hospitales de segundo nivel de atención en Morelia, Michoacán, México.

\section{Metodología}

Estudio descriptivo y transversal ${ }^{(25)}$ realizado en una muestra no probabilística de 160 enfermeras (os) seleccionados a conveniencia a quienes se solicitó su participación en los grupos de Facebook de las investigadoras del estudio. Se incluyeron enfermeras que laboraban en hospitales públicos o privados de segundo nivel de atención de Morelia, Michoacán, México, de ambos sexos, con edad de 18 a 50 años, en cuidado directo a pacientes con COVID-19 o cualquier enfermedad; se excluyeron a enfermeras (os) con licencia por enfermedad o que realizaban actividades administrativas.

Se aplicó una cédula de recolecta de datos de dos partes. La primera sección presentó preguntas para describir a la población con las variables sociodemográficas como edad, sexo, escolaridad, institución, turno, capacitación recibida (lavado de manos, generalidades de la enfermedad COVID-19 y uso de EPP) y material y equipo de protección disponible (toallas de papel, cubrebocas, alcohol gel, careta y EPP). La segunda sección incluyó variables por COVID-19 como curso en línea de la enfermedad COVID-19, contacto con pacientes COVID-19 y reconversión a hospital COVID-19.

La Escala de Incertidumbre ante la enfermedad en versión comunitaria (MUIS-C)(26), consta de 23 ítems, utiliza una escala tipo Likert que va de 1 a 5 , donde $5=$ completamente de acuerdo, y $1=$ no estoy de acuerdo en absoluto. Se suman todos los ítems y se obtiene un puntaje que se clasifica en baja incertidumbre (18 a 42 puntos), moderada incertidumbre ( 43 a 66 puntos) y alta incertidumbre (67 a 90 puntos). Se modificó la escala para dirigirla a enfermeras (os) con énfasis en la enfermedad COVID-19, se realizó una validación de contenido por diez expertos en instrumentos. La versión final modificada consta de 18 ítems, se aplicó a enfermeras de un hospital privado diferente obteniendo una consistencia interna con la prueba alfa de Cronbach de 0.863 .

Estudio aprobado por el Comité de Investigación y Bioética de la Facultad de Enfermería 
(CIB/FacEnf/017/2020). Se realizó colecta de datos en un formulario de Google publicado en la red social Facebook de las investigadoras durante el mes de junio del año 2020, señalando los criterios de selección necesarios para participar, enfatizando que la investigación era sin riesgo y sin costo, el manejo confidencial de los datos, la posibilidad de retractación dado que ninguna pregunta era obligatoria y solicitando expresamente el consentimiento a participar ${ }^{(27)}$. El análisis estadístico se realizó con estadística descriptiva mediante frecuencias, proporciones, medidas de tendencia central y de dispersión. El análisis inferencial fue a través de la prueba Chi-cuadrado de Pearson definiendo un nivel de significancia de $p=<0.05$.

\section{Resultados}

Los participantes tenían una edad promedio de 36.7 años ( $D E=8.5)$, mayoría mujeres (87.5\%), licenciadas en enfermería (63.1\%), pertenecientes a la Secretaría de Salud y laboraban en el turno matutino (37.5\%) (Tabla 1).

Tabla 1. Perfil sociodemográfico de enfermeras de hospitales de segundo nivel de atención, Morelia, Michoacán, México, $2020(n=160)$.

\begin{tabular}{llcc}
\hline Variable & & $f$ & $\%$ \\
\hline \multirow{2}{*}{ Sexo } & Femenino & 140 & 87.5 \\
& Masculino & 20 & 12.5 \\
Escolaridad & Nivel técnico & 27 & 16.9 \\
& Licenciatura & 101 & 63.1 \\
& Posgrado & 32 & 20 \\
Institución & Secretaría de Salud & 108 & 67.5 \\
& IMSS & 24 & 15.0 \\
& ISSSTE & 10 & 6.3 \\
\multirow{3}{*}{ Turno } & Hospital privado & 18 & 11.3 \\
& Matutino & 60 & 37.5 \\
& Vespertino & 31 & 19.4 \\
\hline
\end{tabular}

Fuente: Elaboración propia

IMSS= Instituto Mexicano del Seguro Social; ISSSTE=Instituto de Seguridad y Servicios Sociales de los Trabajadores del Estado.

Respecto a capacitación, $73.1 \%$ de los participantes asistieron a sesiones sobre lavado de manos, 75\% acudieron a sesiones sobre generalidades de la enfermedad COVID-19 y 50.6\% no recibió capacitación 
en el uso de EPP. Las enfermeras (os) tuvieron disponible para el cuidado directo de los personas con COVID19, materiales como toallas de papel (72.5\%), cubrebocas (84.4\%), alcohol gel (68.8\%), caretas (60\%) y un 71.9\% no tuvo acceso a los EPP. Se destaca que $62.5 \%$ de los participantes no se capacitaron con cursos en línea sobre COVID-19, sólo $36.9 \%$ de las enfermeras (os) tuvo contacto con pacientes COVID-19 y 33.8\% de los hospitales fueron reconvertidos a hospital COVID-19 (tabla 2).

Tabla 2. Variables laborales de enfermeras de hospitales de segundo nivel de atención, Morelia, Michoacán, México, $2020(n=160)$.

\begin{tabular}{llcc}
\hline Variables & & $f$ & $\%$ \\
\hline Capacitación & Sí & 117 & 73.1 \\
Lavado de manos & No & 43 & 26.9 \\
Generalidades de COVID-19 & Sí & 120 & 75 \\
Uso de EPP & No & 40 & 25 \\
\hline Disponibilidad de material y equipo & Sí & 79 & 49.4 \\
Toallas de papel & No & 81 & 50.6 \\
& & & \\
Cubrebocas & Sí & 116 & 72.5 \\
Alcohol gel & No & 44 & 27.5 \\
Careta & Sí & 135 & 84.4 \\
& No & 25 & 15.6 \\
EPP & Sí & 110 & 68.8 \\
\hline Variables por COVID-19 & No & 50 & 31.3 \\
Curso en línea enfermedad COVID-19 & Sí & 96 & 60 \\
Contacto con pacientes COVID-19 & No & 64 & 40 \\
Reconversión a hospital COVID-19 & Ní & 45 & 28.1 \\
& No & 115 & 71.9 \\
\hline
\end{tabular}

Fuente: Elaboración propia

En esta muestra, $64.4 \%$ de los participantes presentaron incertidumbre de moderada a alta y sólo 35.6\% mostró baja incertidumbre, (Tabla 3). 
Tabla 3. Incertidumbre en enfermeras de enfermeras de hospitales de segundo nivel de atención, Morelia, Michoacán, México, 2020 ( $n=160)$.

\begin{tabular}{lcc}
\hline Incertidumbre & $f$ & $\%$ \\
\hline Baja & 57 & 35.6 \\
Moderada & 91 & 56.9 \\
Alta & 12 & 7.5 \\
\hline
\end{tabular}

Fuente: Elaboración propia

Cuando se analizó la relación de la incertidumbre con las variables sociodemográficas y laborales, se encontró relación estadísticamente significativa $(p=\leq 0.005)$ entre incertidumbre y las variables sociodemográficas (sexo y escolaridad), en capacitación (generalidades de la enfermedad COVID-19 y uso del EPP), en disponibilidad de material (careta y EPP) y en las variables por COVID-19 (curso en línea COVID19), (tabla 4).

\section{Discusión}

Respecto a las variables sociodemográficas, los participantes de este estudio poseen características similares a otros estudios en China que abordaron los factores asociados a la salud mental del personal expuesto a la enfermedad COVID-19 y el estrés en personal de enfermería, que incluyen ser mayoritariamente mujeres y con grado de licenciatura en enfermería ${ }^{(18-19)}$. Las enfermeras (OS) en atención directa a pacientes de este estudio refirieron tener acceso a toallas de papel para lavado de manos y alcohol gel, pero no al EPP, esta situación ha sido frecuentemente señalada por organismos de enfermería e investigaciones recientes, donde se menciona que el personal de enfermería tienen acceso limitado a los EPP aún en países de primer mundo ${ }^{(12-13)}$, con lo que el riesgo de exposición al virus y la posibilidad de contraer la enfermedad aumenta, lo que puede generar un incremento en el nivel de incertidumbre que enfermeras (os) pueden experimentar durante su trabajo(13, 21-23). 
Tabla 4. Relación entre incertidumbre y variables laborales de hospitales de segundo nivel de atención, Morelia, Michoacán, México, $2020(n=160)$.

\begin{tabular}{|c|c|c|c|c|c|c|c|c|}
\hline \multirow{3}{*}{ Variable } & & \multicolumn{6}{|c|}{ Incertidumbre } & \multirow{3}{*}{$p$} \\
\hline & & \multicolumn{2}{|c|}{ Baja } & \multicolumn{2}{|c|}{ Moderada } & \multicolumn{2}{|c|}{ Alta } & \\
\hline & & $f$ & $\%$ & $f$ & $\%$ & $f$ & $\%$ & \\
\hline \multicolumn{9}{|l|}{ Sociodemográficas } \\
\hline \multirow[t]{2}{*}{ Sexo } & Femenino & 49 & 35 & 83 & 59.3 & 8 & 5.7 & $.049^{* *}$ \\
\hline & Masculino & 8 & 40 & 8 & 40 & 4 & 20 & \\
\hline \multirow[t]{3}{*}{ Escolaridad } & Nivel técnico & 17 & 63 & 9 & 33.3 & 1 & 3.7 & $.014^{\star *}$ \\
\hline & Licenciatura & 31 & 30.7 & 60 & 59.4 & 10 & 9.9 & \\
\hline & Posgrado & 6 & 18.8 & 26 & 81.3 & 0 & 0 & \\
\hline \multicolumn{9}{|l|}{ Capacitación } \\
\hline \multirow[t]{2}{*}{ Lavado de manos } & Sí & 38 & 32.5 & 71 & 60.7 & 8 & 6.8 & .276 \\
\hline & No & 19 & 44.2 & 20 & 46.5 & 4 & 9.3 & \\
\hline \multirow[t]{2}{*}{ Generalidades COVID-19 } & Sí & 28 & 27.5 & 65 & 63.7 & 9 & 8.8 & $.016^{\star \star}$ \\
\hline & No & 31 & 51.7 & 25 & 41.7 & 4 & 6.7 & \\
\hline \multirow[t]{2}{*}{ Uso de EPP } & Sí & 19 & 24.1 & 52 & 65.8 & 8 & 10.1 & $.009^{\star *}$ \\
\hline & No & 38 & 46.9 & 39 & 48.1 & 4 & 4.9 & \\
\hline \multicolumn{9}{|l|}{ Disponibilidad material } \\
\hline \multirow[t]{2}{*}{ Careta } & Sí & 26 & 27.1 & 62 & 64.6 & 8 & 8.3 & $.022^{\star *}$ \\
\hline & No & 31 & 48.4 & 29 & 45.3 & 4 & 6.3 & \\
\hline \multirow[t]{2}{*}{ EPP } & Sí & 9 & 20 & 31 & 68.9 & 5 & 11.1 & $.031^{* *}$ \\
\hline & No & 48 & 41.7 & 60 & 52.2 & 7 & 6.1 & \\
\hline \multicolumn{9}{|l|}{ Variables por COVID-19 } \\
\hline \multirow{2}{*}{ Curso en línea COVID-19 } & Sí & 13 & 21.7 & 43 & 71.7 & 4 & 6.7 & $.011^{\star \star}$ \\
\hline & No & 44 & 44 & 48 & 48 & 8 & 8 & \\
\hline \multirow{2}{*}{ Contacto con pacientes COVID-19 } & Sí & 13 & 24.1 & 35 & 64.8 & 6 & 11.1 & .068 \\
\hline & No & 44 & 24.1 & 56 & 52.8 & 6 & 5.7 & \\
\hline \multirow{2}{*}{ Reconversión a hospital COVID-19 } & Sí & 15 & 25.4 & 40 & 67.8 & 4 & 6.8 & .093 \\
\hline & No & 42 & 41.6 & 51 & 50.5 & 8 & 7.9 & \\
\hline
\end{tabular}

Fuente: Elaboración propia.

${ }^{* *} p=\leq 0.005$

Las enfermeras (os) recibieron capacitación en la institución donde laboraban en temas como lavado de manos y generalidades de la enfermedad COVID-19, pero no en la utilización del EPP; en este sentido, la capacitación es fundamental para las enfermeras (os) cuando se enfrentan a enfermedades emergentes como la causada por el coronavirus SARS-CoV-2, donde los conocimientos se generan día con día. Esto es especialmente importante porque la capacitación del EPP es esencial para garantizar la seguridad de las enfermeras (os), cuando esto no ocurre, el personal de enfermería se siente amenazado por la posibilidad de contagiarse al retirarse el EPP y esto puede ser un factor para la presencia de incertidumbre $(10,13,20-21,24$, 
28-29).

Las enfermeras (os) mayormente no realizaron cursos sobre la COVID-19 disponibles en plataformas del Sistema Nacional de Salud de México. Aunque existen experiencias exitosas en las enfermeras (os) centradas en la capacitación con tasas de infección cero ${ }^{(20)}$, no es lo común, por lo que las instituciones de salud deben garantizar una capacitación necesaria, reciente y basada en evidencia científica. Mishel señala en su teoría que la capacitación y la credibilidad en las autoridades del sistema de salud (fuentes de estructura) provocan la disminución de la incertidumbre ${ }^{(24)}$, lo que sugiere la necesidad de fortalecer el esquema cognitivo del personal de enfermería, es decir, la interpretación subjetiva que hace respecto a la enfermedad para fortalecer el marco de estímulos ${ }^{(26,28)}$.

Respecto al nivel de incertidumbre, más de la mitad de las enfermeras (os) presentaron incertiudmbre de moderada a alta, lo que confirma lo señalado en diversos estudios con el referente teórico de Mishel, donde el marco de los estímulos percibido por el personal de enfermería a través de los estresores personales, laborales o institucionales ${ }^{(8-13)}$ tienen un efecto negativo que provoca la incertidumbre en la

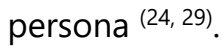

Se observó también que la menor incertidumbre se relacionó en este estudio con tener mayor grado académico, recibir capacitación sobre la enfermedad, el uso de EPP y capacitarse de manera personal con los cursos en línea sobre COVID-19, dado que el conocimiento teórico práctico permite a las enfermeras (o) reconocer sus áreas de mejora e identificar los procesos que aumentan el riesgo de infección como la colocación y extracción de EPP (8-9).

Esto confirma que las fuentes de estructura como la educación o capacitación apoyará la disminución de la incertidumbre en el personal de enfermería ${ }^{(24,26)}$. atención psicológica al personal que se encuentra en primera línea de la enfermedad. 
Como se puede observar, existen diversos factores que provocan incertidumbre en las enfermeras (os) que se encuentran en atención directa a personas con COVID-19 y que requieren que los directivos y las organizaciones de los sistemas de salud tomen medidas para mitigar las secuelas en la salud mental en las enfermeras (os), una de ellas es brindar

\section{Conclusiones}

Dado que el objetivo del estudio fue evaluar el nivel de incertidumbre ante la enfermedad COVID-19 en personal de enfermería de segundo nivel de atención en Morelia, Michoacán, México, se identificó un nivel de incertidumbre de moderado a alto, fue mayor en mujeres y en enfermeras (os) con escolaridad de licenciatura y posgrado; también fue mayor la incertidumbre cuando las enfermeras (os) recibieron capacitación en generalidades de la COVID-19 y el uso de EPP, con disponibilidad de careta y EPP y, finalmente, cuando las enfermeras (os) participaron en cursos en línea COVID-19.

Una limitación de este estudio fue que sólo poco más de un tercio del personal de enfermeras (os) estuvieron en contacto con pacientes con la enfermedad COVID-19, por lo que no se encontró relación estadísticamente significativa entre la incertidumbre y mantener contacto con COVID-19 y la reconversión a hospital COVID-19. Otras limitaciones para señalar son el empleo de una muestra no probabilística y un diseño transversal que no considera los cambios que pueden experimentar las enfermeras (os) a lo largo de la epidemia. Este estudio permite un acercamiento a la realidad que vivieron las enfermeras (os) de México durante la atención a la población durante la pandemia por la enfermedad COVID-19, por lo que se se espera que los resultados permitan a los directivos de enfermería en México proponer intervenciones personalizadas que mejoren la salud mental de su personal ante situaciones de incertidumbre que experimentan de forma cotidiana.

\section{Conflicto de intereses}

Los autores declaran no tener conflicto de intereses. 


\section{Financiamiento}

Esta investigación no tuvo financiamiento para su realización.

\section{Referencias bibliográficas}

1. Organización Mundial de la Salud. COVID-19: Cronología de la actuación de la OMS. Geneva: OMS [Internet]. 2020 [citado 10 jun 2020]. Disponible en: https://www.who.int/news-room/detail/27-04-2020who-timeline---covid-19

2. International Council of Nurses. Evidence-based safe nurse staffing. Position Statement. Geneva: ICN [Internet]. 2018 [citado 20 jun 2020]. Disponible en: https://www.icn.ch/sites/default/files/inlinefiles/PS C \%20Evidence\%20based\%20safe\%20nurse\%20staffing 1.pdf

3. World Health Organization. State of the world's nursing 2020: executive summary. Geneva: WHO [Internet]. 2020 [citado 20 jun 2020]. Disponible en: https://apps.who.int/iris/handle/10665/331673

4. Subsecretaría de Integración y Desarrollo del Sector Salud. Estado de la enfermería en México [Internet]. 2018 [citado jun 2020]. Disponible en: http://www.cpe.salud.gob.mx/site3/publicaciones/docs/estado enfermeria mexico2018.pdf

5. Aldrete MG, González R, Navarro C, León C, Pérez SG. Nursing staff psychosocial factors and stress in nursing professionals of a third-level public hospital of care. Rev Salud Publica Nutr [Internet]. 2016 [citado 10 jun 2020];15(3):8-15. Disponible en: http://respyn.uanl.mx/index.php/respyn/article/view/14

6. Freitas TL, Banazeski AC, Eisele A, De Souza EN, Bitencourt JV, Souza S. La visión de la enfermería ante el proceso de muerte y morir de pacientes críticos: una revisión integradora. Enferm Glob [Internet]. 2016 [citado 20 jun 2020];15(41):322-334. Disponible en: http://scielo.isciii.es/scielo.php?script=sci arttext\&pid=S1695-61412016000100015\&lng=es\&tlng=es

7. Pan American Health Organization. MHPSS for Health Professionals Involved in the COVID-19 Response. Washington, DC: Oficina Regional para las Américas de la Organización Mundial de la Salud [Internet]. 2020 [citado 20 jun 2020]. Disponible en:

8. Petzold MB, Plag J, Ströhle A. Umgang mit psychischer belastung bei gesundheitsfachkräften im rahmen der Covid-19-Pandemie. Nervenarzt [Internet]. 2020 [citado 20 jun 2020];91:417-421. Disponible en: https://doi.org/10.1007/s00115-020-00905-0

9. Nayna-Schwerdtle P, Connell C, Lee S, Plummer V, Russo P, Endacott R, et al. Nurse expertise: a critical resource in the COVID-19 pandemic response. Annals of Global Health [Internet]. 2020 [citado 20 jun 2020];86(1):1-5. Disponible en: https://doi.org/10.5334/aogh.2898

10. Shen $X$, Zou X, Zhong X, Yan J, Li L. Psychological stress of ICU nurses in the time of COVID-19. Critical Care [Internet]. 2020 [citado 20 jun 2020];24:1-3. Disponible en: https://doi.org/10.1186/s13054-020-02926$\underline{2}$

11. Ornell F, Halpern S, Kessler FH, Narvaez J. The impact of the COVID-19 pandemic on the mental health of healthcare professionals. Cadernos de Saúde Pública [Internet]. 2020 [citado 20 jun 2020];36(4):1-6. Disponible en: https://doi.org/10.1590/0102-311X00063520

12. Delgado D, Wyss F, Pérez G, Sosa A, Ponte-Negretti C, Mendoza I, et al. Personal safety during the COVID-19 Pandemic: Realities and perspectives of healthcare workers in Latin America. Int J Environ Res 
Public Health [Internet]. 2020 [citado 20 jun 2020];17:1-8. Disponible en: https://doi.org/10.3390/ijerph17082798

13. Houghton $C$, Meskell P, Delaney $H$, Smalle M, Glenton C, Booth $A$, et al. Barriers and facilitators to healthcare workers' adherence with infection prevention and control (IPC) guidelines for respiratory infectious diseases: a rapid qualitative evidence synthesis. Cochrane database of systematic reviews [Internet]. 2020 [citado 20 jun 2020]; 4:1-57. Disponible en: http:/doi.org/10.1002/14651858.CD013582

14. Lake E. How effective response to COVID-19 relies on nursing research. Res Nurs Health [Internet]. 2020 [citado 20 jun 2020];43:213-214. Disponible en: http://doi.og/10.1002/nur.22025

15. Garza R, Meléndez MC, Castañeda-Hidalgo H, Aguilera A, Acevedo G, Rangel S. Nivel de estrés en enfermeras que laboran en áreas de hospitalización. Desarrollo Cientif Enfer [Internet]. 2011 [citado 20 jun 2020];19(1):15-19. Disponible en: http://www.index-f.com/dce/19pdf/19-015.pdf

16. De Andrés-Gimeno B, Solís-Muñoz M, Revuelta-Zamorano $M$, Sánchez-Herrero $H$, Santano-Magariño A, Grupo de Cuidados COVID-19 HUPHM. Nursing care for hospitalized patients in COVID-19 units. Enferm Clin [Internet]. 2021 [citado 10 mar 2021];31(Supl 1):S49-S54. Disponible en: https://doi.org/10.1016/j.enfcli.2020.05.016

17. Hill B. The COVID-19 pandemic. British Journal of Nursing [Internet]. 2020 [citado 20 jun 2020];29(8):456. Disponible en: $\underline{\text { https://doi.org/10.12968/bjon.2020.29.8.456 }}$

18. Lai J, Ma S, Wang Y, Cai Z, Hu J, Wei N, et al. Factors associated with mental health outcomes among health care workers exposed to coronavirus disease 2019. JAMA Netw Open [Internet]. 2020 [citado 20 jun 2020];3(3):1-12. Disponible en: http://doi.og/10.1001/jamanetworkopen.2020.3976

19. Mo Y, Deng L, Zhang L, Lang Q, Liao Ch, Wang N, et al. Work stress among chinese nurses to support Wuhan in fighting against COVID-19 epidemic. J Nurs Manag [Internet]. 2020 [citado 20 jun 2020];00:1-8. Disponible en: http://doi.org/10.1111/jonm.13014

20. Huang L, Lin G, Tang L, Yu L, Zhou Z. Special attention to nurses' protection during the COVID-19 epidemic. Critical Care [Internet]. 2020 [citado 20 jun 2020];24(120):1-3. Disponible en: https://doi.org/10.1186/s13054-020-2841-7

21. Millar RC. Nursing a patient with Covid-19 infection. J Evidence-based Nurs Prac [Internet]. 2020 [citado 20 jun 2020];1(1):4-8. Disponible en: https://journal-ebnp.com/files/2020/02/nursing a patient with covid19 infection.pdf

22. Chen SC, Lai YH, Tsay SL. Nursing perspectives on the impacts of COVID-19. J Nurs Res [Internet]. 2020 [citado 20 jun 2020];28(3):1-5. Disponible en: https://doi.org/10.1097/NRJ.0000000000000389

23. Maben J, Bridges J. Covid-19: Supporting nurses' psychological and mental health. J Clin Nurs [Internet]. 2020 [citado 20 jun 2020];00:1-9. Disponible en: http://doi.org/10.1111/jocn.15307

24. Bailey DE, Stewart JL. Teoría de la incertidumbre frente a la enfermedad. En: Raile-Alligood M. Modelos y teorías en enfermería. $9^{a}$ ed. Barcelona: Elsevier. 2018. p. 1189-1230.

25. Grove S, Gray J. Investigación en enfermería. Desarrollo de la práctica enfermera basada en la evidencia. $7^{\text {a }}$ ed. Barcelona: Elsevier; 2019. p. 192-226.

26. Torres-Ortega C, Peña-Amaro P. Cross-cultural adaptation of the Mishel uncertainty in illness scale, in a population with chronic kidney disease treated with hemodialysis. Enferm Clin [Internet]. 2015 [citado 20 jun 2020];25(1):15-18. Disponible en: http://dx.doi.org/10.1016/j.enfcli.2014.09.002

27. Diario Oficial de la Federación. Reglamento de la Ley General de Salud en Materia de Investigación para la Salud de México. [Internet]. México: Cámara de Diputados H. Congreso de la Unión; 2014. [citado 20 jun 
2020]. Disponible en: $\underline{\text { http://www.diputados.gob.mx/LeyesBiblio/regley/Reg LGS MIS.pdf }}$

28. Hill B. The COVID-19 pandemic. British Journal of Nursing [Internet]. 2020 [citado 20 jun 2020];29(8):456.

Disponible en: https://doi.org/10.12968/bjon.2020.29.8.456

29. Smith G, Li W. COVID-19: Emerging compassion, courage and resilience in the face of misinformation and adversity. J Clin Nurs [Internet]. 2020 [citado 20 jun 2020];29:1425-1428. Disponible en: http://doi.org/10.1111/jocn.15231

30. International Council of Nurses. More than 600 nurses die from COVID-19 worldwide. Geneva: ICN [Internet]. 2020. [citado 20 jun 2020]. Disponible en: https://www.icn.ch/news/more-600-nurses-die-covid19-worldwide

31. Raurell-Torredá M. Management of ICU nursing teams during the COVID-19 pandemic. Enferm Intensiva [Internet]. 2020 [citado 20 jun 2020];31(2):49-51. Disponible en: http://doi.gob/10.1016/j.enfi.2020.04.001 32. International Council of Nurses. International Council of Nurses highlights top priorities to beat COVID19. Press information. Geneva: CIE [Internet]. 2020. [citado 20 jun 2020] . Disponible en: https://www.icn.ch/system/files/documents/2020-04/ICN\%20briefing COVID19 Top priorities ENG.pdf

Cómo citar este artículo: Ruiz-Recéndiz $M$, Jiménez-Arroyo $V$, Huerta-Baltazar $M$, Alcántar-Zavala $M$, Herrera-Paredes J, González-Villegas G. Incertidumbre ante la enfermedad COVID-19 en personal de

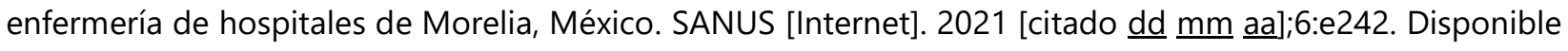
en: URL/DOI 